\title{
Miocarditis aguda en varón de 22 años
}

\author{
Acute myocarditis in 22 year-old man
}

\author{
ML. Valle Feijóo ${ }^{1}$, C. Delgado Sánchez-Gracián², M. Sanmartín Fernández ${ }^{3}$, J. de la Fuente Aguado ${ }^{1}$. \\ ${ }^{1}$ Servicio de Medicina Interna, ${ }^{2}$ Servicio de Radiología. ${ }^{3}$ Servicio de Cardiología. Hospital Povisa. Vigo
}

\begin{abstract}
Resumen
La miocarditis es una enfermedad inflamatoria del miocardio. La infección viral es la causa más común de miocarditis en países desarrollados y puede presentarse con una amplia gama de síntomas, desde disnea o dolor torácico que se resuelve sin tratamiento específico a shock cardiogénico y muerte. El diagnóstico presuntivo se basa en la presentación clínica y pruebas diagnósticas no invasivas, aunque la biopsia endomiocárdica continua siendo el patrón de oro para el diagnóstico in vivo de miocarditis. Tras su implantación en la práctica clínica, la resonancia cardíaca se ha convertido en la herramienta principal para la evaluación no invasiva de inflamación del miocardio en pacientes con sospecha de miocarditis.

Describimos el caso de un joven que fue admitido por dolor torácico y disnea aguda, donde la resonancia cardíaca fue la herramienta diagnóstica fundamental para el diagnóstico de miocarditis.

Palabras clave: Miocarditis. Resonancia magnética cardíaca.
\end{abstract}

\section{Introducción}

La miocarditis es un proceso inflamatorio que afecta al miocardio en respuesta a diversos agentes infecciosos, químicos y/o físicos. Afecta predominantemente a niños y adultos jóvenes y los síntomas suelen ser inespecíficos. La mayoría de los pacientes presentan recuperación completa espontánea, sin embargo en ocasiones pueden evolucionar a miocardiopatía dilatada o incluso a la muerte.

El diagnóstico se realiza clínicamente y por métodos de imagen no invasivos, indicándose la biopsia endomiocárdica sólo en casos seleccionados. En los últimos años, la resonancia magnética cardíaca (RMC) ha emergido como una herramienta diagnóstica muy útil.

\section{Caso clínico}

Varón de 22 años, fumador de 5 cigarrillos/día, sin historia de consumo de drogas ni factores de riesgo cardiovascular conocidos, que acude a urgencias por dolor centrotorácico intenso, que aumentaba con la inspiración, acompañado de disnea aguda de 3 horas de evolución. Refería además sensación distérmica, náuseas y vómitos en las últimas 24 horas. A su llegada a urgencias presentaba TA 130/70 mmHg, FC $126 \mathrm{Ipm}$ y $\mathrm{T}^{\mathrm{a}} 38.4^{\circ} \mathrm{C}$. La auscultación cardíaca era normal y en la auscultación pulmonar presentaba hipoventilación bibasal con crepitantes bilaterales dispersos; el resto de la exploración era anodina. En el electrocardiograma se apreciaba taquicardia sinusal e inversión de la onda T de V4 a V6. Presentaba además leucocitosis (leucocitos: $14.47 \times 10^{3} / \mathrm{mm}^{3}$ ) con normalidad del resto de parámetros hematológicos, bioquímicos y del estudio de coagulación. Los marcadores de necrosis miocárdica se encontraban elevados

\begin{abstract}
Myocarditis is an inflammatory disease of the myocardium. Viral infection is the most common cause of myocarditis in developed countries and it may present with a wide range of symptoms, ranging from dyspnea or chest pain that resolves without specific therapy to cardiogenic shock and death. The diagnosis is made based on clinical signs and symptoms and noninvasive diagnostic tests, but the endomyocardial biopsy remains the gold standard for in vivo diagnosis of myocarditis. Following its general accessibility in clinical practice, cardiovascular magnetic resonance imaging has become the key tool for noninvasive assessment of myocardial inflammation in patients with suspected myocarditis.

We report a case of a young adult who was admitted for chest pain and acute dyspnea, and where the cardiovascular magnetic resonance was the key tool for the diagnosis of myocarditis.

Key words: Myocarditis. Cardiovascular magnetic resonance.
\end{abstract}

(CPK 682 U/L; Mioglobina: 157 ng/mL; Troponina I: 20 ng/mL) y la radiografía de tórax reveló un infiltrado intersticial bilateral. Posteriormente el paciente sufrió inestabilidad hemodinámica, taquipnea y desaturación, decidiéndose ingreso en la Unidad de Cuidados Intensivos. La TAC torácica mostró edema intersticial, derrame pleural bilateral y cardiomegalia a expensas de crecimiento del ventrículo izquierdo, todo ello compatible con fallo cardíaco. El ecocardiograma reveló hipocinesia global del ventrículo izquierdo con fracción de eyección (FE) del 30-35\%, sin otras alteraciones a nivel del ventrículo derecho, válvulas o pericardio. La evolución clínica fue favorable con diurético e inhibidor de la enzima de conversión de la angiotensina, lo que permitió su traslado a planta a las 48 horas.

Figura 1. Resonancia Magnética Cardíaca realizada durante el ingreso. A) Secuencia STIR en el plano del eje corto a nivel apical donde se observa un aumento de intensidad de la señal en la región anteroseptal (flecha hueca), que indica edema miocárdico. B) Secuencia de realce tardío en el plano de las cuatro cámaras que muestra áreas de hiperrealce parcheadas y subepicárdicas en la pared lateral del ventrículo izquierdo (flechas) reflejando necrosis tisular.

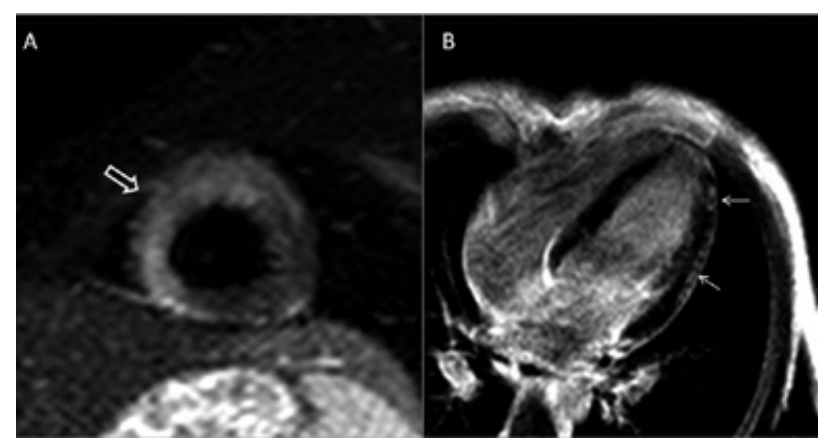


Tabla 1. Etiología de la miocarditis

\begin{tabular}{|c|c|}
\hline Infecciones & 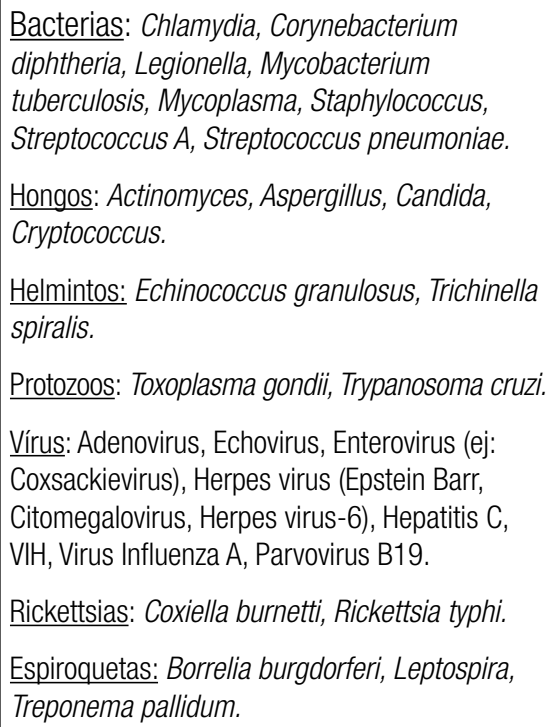 \\
\hline Enf. Autoinmunes & $\begin{array}{l}\text { Enfermedad celíaca, Síndrome de } \\
\text { Churg-Strauss, Enfermedad de Crohn, } \\
\text { Dermatomiosistis, Miocarditis de células } \\
\text { gigantes, Síndrome Hipereosinofílico, Enf. de } \\
\text { Kawasaki, LES, Miocarditis Linfofolicular, Artritis } \\
\text { Reumatoide, Sarcoidosis, Esclerodermia, Colitis } \\
\text { Ulcerosa }\end{array}$ \\
\hline $\begin{array}{l}\text { Reacciones de } \\
\text { hipersensibilidad }\end{array}$ & $\begin{array}{l}\text { Penicilina, Ampicilina, Cefalosporinas, } \\
\text { Tetraciclinas, Sulfonamidas, Antiflogísticos, } \\
\text { Benzodiacepinas, Clozapina, Diuréticos de asa, } \\
\text { Metildopa, Vacuna de la viruela, Toxoide tetánico, } \\
\text { Antidepresivos tricíclicos }\end{array}$ \\
\hline $\begin{array}{l}\text { Reacciones } \\
\text { tóxicas }\end{array}$ & $\begin{array}{l}\text { Anfetaminas, Antraciclinas, Catecolaminas, } \\
\text { Cocaína, Ciclofosfamida, 5-fluoracilo, Fenitoína, } \\
\text { Trastuzumab }\end{array}$ \\
\hline Tóxicos & Etanol \\
\hline Otros & Arsénico, cobre, hierro, radioterapia, tirotoxicosis \\
\hline
\end{tabular}

Con el fin de aclarar el diagnóstico se realizó una RMC en la que presentaba hiperseñal en secuencias STIR (Short-Tau Inversión Recovery), afectando a la región del ápex, septo interventricular y pared lateral del ventrículo izquierdo así como áreas de realce tardío parcheadas sin patrón de distribución vascular en la pared lateral del ventrículo izquierdo. Estos hallazgos fueron compatibles con miocarditis aguda (Figura 1). A las 6 semanas el paciente se encontraba asintomático y tanto el ecocardiograma como la RMC de control mostraron un ventrículo izquierdo de morfología, tamaño y contractilidad global y segmentaria conservadas con FE del $69 \%$ así como mejoría de los signos de miocarditis aguda, persistiendo realce tardío en la pared lateral del ventrículo izquierdo (Figura 2).

\section{Discusión}

La miocarditis es un proceso inflamatorio producto de la agresión miocárdica de un amplio espectro de patógenos infecciosos, que incluyen virus, bacterias, hongos y protozoos, así como reacciones tóxicas y de hipersensibilidad ${ }^{1}$ (Tabla 1)². En los países desarrollados la infección vírica
Figura 2. Resonancia Magnética cardíaca de control realizada 6 semanas más tarde. A) Secuencia STIR en el plano del eje corto a nivel apical donde se observa una mejoría del edema miocárdico al desaparecer la intensidad de señal anteroseptal. B) Secuencia de realce tardío en el plano de las cuatro cámaras que continúa mostrando las áreas de hiperrealce parcheadas y subepicárdicas en la pared lateral del ventrículo izquierdo (flechas) indicando que persiste la necrosis tisular del miocardio.

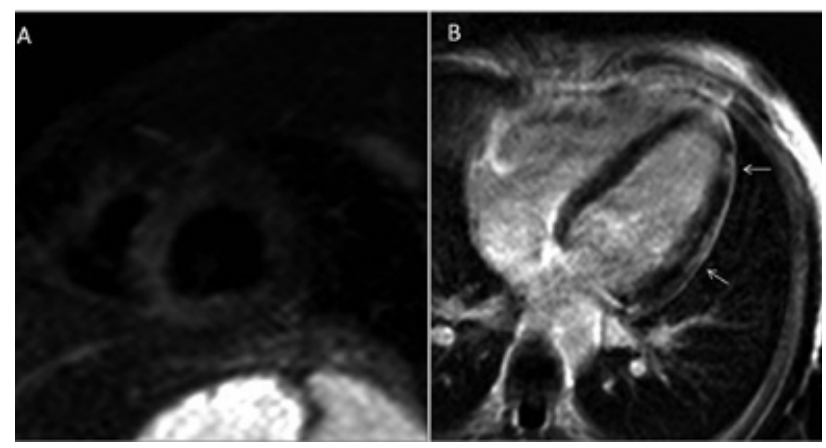

es la más frecuentemente implicada. Si bien hasta la década de los 90, los enterovirus (Coxsackie B) eran los virus más prevalentes, en la actualidad gracias al desarrollo de técnicas como la reacción en cadena de la polimerasa (PCR) e hibridación in situ, el espectro de virus encontrados en las biopsias endomiocárdicas (BEM) ha variado², cobrando relevancia el parvovirus y el herpesvirus humano 6. No obstante los enterovirus continúan siendo una causa importante de miocarditis en determinadas regiones ${ }^{1}$. Puede afectar a cualquier cámara cardíaca de forma focal o difusa. Hay un discreto predominio del sexo masculino, que se ha atribuido al efecto protector de las hormonas femeninas sobre la inmunomodulación ${ }^{3}$. Su presentación clínica en adultos es ampliamente variable, desde alteraciones electrocardiográficas asintomáticas hasta shock cardiogénic $0^{3}$. Los pródromos virales oscilan entre un 10 y un $80 \%$ de los casos según las series y comprenden fiebre, mialgias, síntomas respiratorios y gastrointestinales ${ }^{4}$. Los síntomas cardíacos son variables siendo la disnea el más frecuente (72\% de los casos), sobre todo en pacientes con disfunción ventricular, seguido de dolor torácico (32\%) y episodios arrítmicos $(18 \%)^{5}$. Muchas veces se presenta como una miocardiopatía dilatada de etiología no isquémica en pacientes que habían presentado síntomas pocas semanas 0 meses antes ${ }^{6}$; de hecho, en el seguimiento de la miocarditis a los tres años se constató que hasta un $21 \%$ de los casos desarrollaban una miocardiopatía dilatada ${ }^{7}$, siendo esta a su vez, uno de los motivos más frecuentes de trasplante cardíaco². En algunos casos la miocarditis aguda puede producir muerte súbita, como expresión de un bloqueo completo 0 , más habitualmente, de una taquicardia 0 fibrilación ventricular. En estudios post mórtem de jóvenes fallecidos por muerte súbita, el porcentaje de miocarditis llegó a alcanzar el 8.6-12\%8.

Como consecuencia de la gran variabilidad clínica y de la ausencia de métodos diagnósticos capaces de garantizar 
un grado de especificidad y sensibilidad aceptable (biomarcadores, electrocardiograma, ecocardiograma, serologías y estudios isotópicos), la miocarditis hasta hace pocos años suponía un reto diagnóstico y, probablemente por ello, se encontraba infradiagnosticada. Si bien la BEM fue el método diagnóstico de referencia y actualmente es la técnica que otorga el diagnóstico definitivo, presenta varios inconvenientes como baja sensibilidad, gran variabilidad interobservador en el proceso de interpretación de las muestras histopatológicas y una morbi-mortalidad no despreciable. En 2007, la American Heart Association, el American College of Cardiology y la European Society of Cardiology recomendaron la BEM en dos escenarios clínicos: miocardiopatía dilatada rápidamente progresiva refractaria al tratamiento o miocardiopatía de causa desconocida con arritmias que amenazan la vida9 ${ }^{9}$ A pesar de ello, la BEM con análisis inmunohistoquímico y técnicas de biología molecular tiene cada vez más un papel relevante en el diagnóstico de las miocarditis y estudios recientes apuntan también a su importancia en la valoración pronóstica ${ }^{10}$.

En este contexto, durante la última década han surgido múltiples estudios en los que la RMC ha emergido como una técnica no invasiva segura, que permite valorar la función cardíaca y el daño miocárdico en pacientes con alta probabilidad clínica de miocarditis ${ }^{11}$. Permite estudiar marcadores de lesión tisular, detectando edema tisular (secuencias potenciadas en T2, STIR), hiperemia (secuencias potenciadas en $\mathrm{T} 1$ con y sin gadolinio para estudiar el realce precoz) y lesión miocárdica irreversible (secuencias de realce tardío) ${ }^{12}$ visualizando áreas de hiperrealce causadas por el contraste acumulado en el miocárdico como consecuencia de la desestructuración de la membrana miocitaria ocasionada por el proceso inflamatorio. Además permite determinar volúmenes, fracción de eyección ventriculares y derrame pleuropericárdico. En un consenso reciente del International Consensus Group on Cardiovascular Magnetic Resonance in Myocarditis los autores recomiendan la adquisición de las 3 secuencias de caracterización tisular previamente citadas (STIR para edema, realce precoz para hiperemia y realce tardío para lesión miocárdica irreversible) $)^{13}$. La combinación de las secuencias aumenta la precisión diagnóstica. Si son positivas 20 más la precisión diagnóstica es del $78 \%$, la sensibilidad del $76 \%$ y la especificidad del $96 \%$. Si solo se utiliza la secuencia del realce tardío la precisión diagnóstica es del 68\% con una sensibilidad del $44-54 \%$ y una especificidad del $60-100 \%{ }^{13}$.

La RMC con contraste puede ser utilizada para distinguir entre procesos agudos y crónicos ${ }^{11}$, en la monitorización de la evolución ${ }^{12,14}$ y como guía de la BEM hacia zonas de realce $^{14}$. La RMC no solo es una herramienta diagnóstica, sino que puede aportar valor pronóstico. Así, Wagner et al. en 2003 demostraron que la persistencia del realce precoz de gadolinio a partir de las 4 semanas del inicio de la clínica se asocia a un peor pronóstico en cuanto a la recuperación funcional y la persistencia de síntomas en el control a los 3 años ${ }^{15}$. En un estudio reciente de Grün et al. que incluyó pacientes con miocarditis viral diagnosticada por biopsia, la presencia de realce tardío en la RMC resultó ser el mejor predictor independiente de mortalidad global y cardiaca a los 5 años ${ }^{16}$.

En el paciente que se presenta, la demostración de la afectación inflamatoria del miocardio en secuencias STIR y el patrón de distribución de gadolinio permitieron establecer el diagnóstico de miocarditis aguda. Estos signos de miocarditis aguda habían mejorado en la RMC de seguimiento, persistiendo realce tardío en la pared lateral del ventrículo izquierdo.

En resumen, aunque la BEM con estudio inmunohistoquímico sigue siendo la técnica de referencia para establecer un diagnóstico definitivo de miocarditis, la RMC por su carácter no invasivo y por la información morfológica, funcional y de caracterización tisular que ofrece sea probablemente en el futuro inmediato el método diagnóstico de elección.

\section{Bibliografía}

1. Sagar S, Liu PP, Cooper LT Jr. Myocarditis. Lancet 2012; 379:738-47.

2. Kindermann I, Barth C, Mahfoud F et al. Uptodate in Myocarditis. J Am Coll Cardiol 2012;59:779-92.

3. Blauwet LA, Cooper LT. Myocarditis. Prog Cardiovasc Dis. 2010;52(4):274-88.

4. Magnani JW, Dec GW. Myocarditis: Current trends in diagnosis and treatment. Circulation 2006;113:876-90.

5. Hufnagel G, Pankuweit S, Richter A, et al., for the ESETCID Investigators. The European Study of Epidemiology and Treatment of Cardiac Inflammatory Diseases (ESETCID): First epidemiological results. Herz. 2000;25:279-85.

6. Cooper LT Jr. Myocarditis. N Engl Med 2009; 360: 1526-38.

7. D’Ambrosio A, Patti G, Manzoli A et al. The fate of acute myocarditis between spontaneous improvement and evolution to dilated cardiomyopathy: a review. Heart 2001; 85: 499-504.

8. Fabre A., Sheppard MN. Sudden adult death syndrome and other non ischaemic causes of sudden cardiac death. Heart 2006; 92:316-20.

9. Cooper LT, Baughman KL, Feldman AM, et al. The role of endomyocardial biopsy in the management of cardiovascular disease: a scientific statement from the American Heart Association, the American College of Cardiology, and the European Society of Cardiology. Circulation 2007;116: 2216-33.

10. Kindermann I, Kindermann M, Kandolf R, et al. Predictors of outcome in patients with suspected myocarditis. Circulation 2008;118:639-48.

11. Childs H, Friedrich MG. Cardiovascular Magnetic Resonance Imaging in Myocarditis. Prog Cardiovasc Dis 2011;54:266-75.

12. Friedrich MG, Strohm 0, Schulz-Menger J, et al. Contrast media-enhanced magnetic resonance imaging visualizes myocardial changes in the course of viral myocarditis. Circulation 1998;97:1802-1809.

13. Friedrich MG, Sechtem U, Schulz-Menger J, et al. Cardiovascular magnetic resonance in myocarditis: a JACC White Paper. J Am Coll Cardiol 2009;53: 1475-87.

14. Mahrholdt H, Goedecke C, Wagner A, Meinhardt G, et al. Cardiovascular magnetic resonance assessment of human myocarditis: a comparison to histology and molecular pathology. Circulation 2004; 109:1250-58.

15. Wagner A, Schulz-Menger J, Dietz R, Friedrich MG. Long-term follow-up of patients with acute myocarditis by magnetic resonance imaging. MAGMA 2003;16:17-20.

16. Grün S, Schumm J, Greulich S, et-al. Long-term follow-up of biopsy proven viral myocarditis: Predictors of mortality and incomplete recovery. J Am Coll Cardiol. 2012; 59:1604-15 